Editorial

\title{
Acknowledgement to Reviewers of Minerals in 2017
}

\author{
Minerals Editorial Office \\ MDPI AG, St. Alban-Anlage 66, 4052 Basel, Switzerland \\ Published: 19 January 2018
}

Peer review is an essential part in the publication process, ensuring that Minerals maintains high quality standards for its published papers. In 2017, a total of 252 papers were published in the journal. Thanks to the cooperation of our reviewers, the median time to first decision was 19 days and the median time to publication was 48 days. The editors would like to express their sincere gratitude to the following reviewers for their time and dedication in 2017:

Abella, Pura Alfonso

Addai-Mensah, Jonas

Afanasiev, P.

Agnieszka, Gruszecka-Kosowska

Agorhom, Eric Aminartey

Aines, Roger D.

Akar, Gul

Akbari, Hamid

Alagha, Lana

Alehossein, Habib

Allen, Charlotte

Almeida, José António De

Alonzo D., Cook

Alorro, Richard Diaz

Amankwah, Anthony

Amato, Alessia

Amenabar, Maximiliano

Amils, Ricardo

Andersen, Jens

Andrä, Heiko

Anthony, Ben

Aparicio, Conrado

Apopei, Andrei Ionut

Arbuzov, S.I.

Ardakani, Omid

Ardit, Matteo

Arena, Derrick Adam

Arias, José L.

Ashekuzzaman, S.M.
Atkins, Anthony S.

Augustsson, Carita

Aureliano, Manuel

Awe, Samuel

Ayele, Yonas Zewdu

Azevedo, Leonardo

B. Larsen, Rune

Bach, Vanessa

Badenhorst, Heinrich

Bakker, Ronald

Balassone, Giuseppina

Balomenos, Thymis

Baltakys, Kestutis

Bark, Glenn

Bas, Ahmet Deniz

Bascompta, Marc

Bazin, Glaude

Beck, Andrew

Bédard, Paul

Belviso, Claudia

Bender, Kelly

Benison, Kathy

Benito, José Manuel

Benzerara, Karim

Berry, Ron

Bhattacharyya, Amrita

Billström, Kjell

Bindi, Luca

Bindschedler, Saskia 
Blanchard, Peter E.R.

Blazquez, Maria Luisa

Block, Karin

Bobadilla Fazzini, Roberto

Bobicki, Erin R.

Boehm, Andreas

Bontempi, Elza

Borkiewicz, Olaf

Borra, Chenna Rao

Bosse, Valerie

Bournival, Ghislain

Bouropoulos, Nikolaos

Bowles, John

Boxall, Naomi

Brauer, Suzanna

Brendlé, Jocelyne

Brezáni, Ivan

Britvin, Sergey N.

Brueckner, Stefanie

Buckman, Jim

Budzyn, Bartosz

Burat, Firat

Çabalar, Ali Firat

Cabedo, Luis

Campbell, Andrew R.

Carmicahel, Sarah

Case, George

Castellini, Elena

Castilla-Gómez, Jorge

Castro, Laura

Castro-Gomes, João

Cavalazzi, Barbara

Cavalline, Tara

Cecilia, Juan Antonio

Cejka, Jiri

Cepedal, Antonia

Chapman, Robert

Chaumba, Jeff

Chauviré, Boris

Chelgani, Saeed Chehreh

Chen, Pao-Chi
Chen, Cheng

Cheng, Liang

Cheong, Chang-Sik

Chessa, Manuela

Chiozzi, Paolo

Chivers, Tristram

Choi, Siyoung Q.

Choi, Heekyu

Chopard, Aurélie

Choulet, Flavien

Christanis, Kimon

Christiansen, Eric

Christy, Andrew G.

Ciceri, Davide

Ciesielczuk, Justyna

Cilliers, Jan

Cisternas, Luis A.

Cohen, David

Colomban, Philippe

Connor, Jason

Cook, Nigel

Corin, Kirsten Claire

Costin, Gelu

Courtin-Nomade, Alexandra

Coveney, Raymond

Cristelo, Nuno Miguel

Crivelli, Davide

Crocket, Kirsty

Dai, Zongfu

Danko, George

Davide, Mombelli

Day, Warren C.

De Giudici, Giovanni

Debaille, Vinciane

Dedzo, Gustave Kenne

Del Hoyo, C.

Delle Rose, Marco

Demers, Isabelle

Demichelis, Raffaella

Deniz, Vedat

Di, Haibin 
Di Palma, Luca

DiBenedetto, Francesco

Dinis, Maria De Lurdes Proença De Amorim

Dlugogorski, Bogdan Z.

Dobrzhinetskaya, Larissa F.

Dominguez-Ramos, Antonio

Donatello, Shane

Dorado Castaño, Antonio David

Dostal, Jaroslav

Dragovic, Besim

Drüppel, Kirsten

Drzymala, Jan

Dubrule, Olivier

Duchesne, Jean-Clair

DuFrane, Scott Andrew

Dumańska-Słowik, Magdalena

Dyer, Laurence G.

Eble, Cortland

Edraki, Mansour

Ehrig, Kathy

Einarsrud, Kristian Etienne

Ejtemaei, Majid

Eliasson, Veronica

Elliott, Brent

Elmi, Chiara

Elmi Assadzadeh, Golpira

Elsen, Jan

Emad, Muhammad Zaka

Emery, Xavier

Erastova, Valentina

Ercikdi, Bayram

Eric, Franz

Esmaeili, Kamran

Espinoza-Marzal, Rosa

Estokova, Adriana

Etschmann, Barbara

Exadaktylos, George

Eyre, Thomas

Faheem, Ahmed F.

Faraci, Francesca Dalia

Farrokhpay, Saeed
Faybishenko, Boris A.

Feng, Qicheng

Fernández, Raúl

Fernández-Díaz, Lourdes

Fernandez-Martinez, Alejandro

Finkelman, Robert

Finneran, Kevin

Flores, Deolinda

Fornasiero, Daniel

Forsberg, Kerstin

Forstner, Stephanie

Fouedjio, Francky

Fowdar, Harsha

Franz, Gerhard

Frenzel, Max

Frost, Ray

Fujita, Toyohisa

Fulton, Mike

Gabitov, Rinat

Gaillou, Eloise

Galuskin, Evgeny

Gamage, Ranjith

Gan, Quan

Gao, Zhiye

Gao, Xiaodong

Garcia, Rosario

García-Veigas, Javier

Garg, Nishant

Gartman, Amy

Gattacceca, Jérôme

Gazley, Michael

Gázquez, Fernando

Geatches, Dawn

Gee, Carol

Gentina, Juan Carlos

Genty, Eric

Ghirian, Alireza

Ghorbani, Yousef

Gianni, Areti

Glasspool, Ian

Godlewska-Żyłkiewicz, Beata 
Golla-Schindler, Ute Maria

Golokhvast, Kirill

Gomari, Sina Rezaei

Gonzalez-Nicolas, Ana

Goryachev, B.E.

Gregory, Daniel D.

Guil, José María

Guldris, Lorena

Gunasekara, Chamila

Guo, Xiaofeng

Gupta, Nikhil

Gutzmer, Jens

Gyorgy, Czuppon

Habashi, Fathi

Haddadi, Farid

Hagen-Peter, Graham

Hammarstrom, Jane

Harlov, Daniel

Harrison, Anna

Hartlieb, Philipp

Hasan, Maruf

Hathon, Lori

He, Hongpin

Hebblewhite, Bruce

Hedin, Niklas

Henry, Chris

Hernandez, Eduardo R.

Hirajima, Tsuyoshi

Hiroshi, Sakuma

Hiskey, J. Brent

Hitzman, Murray

Hjertager, Bjørn

Hoagland, Porter

Holmes, Ralph

Holuszko, Maria

Hopkinson, Laurence

Horner, Olivier

Hower, Jim

$\mathrm{Hu}$, Allen

Huang, Qingqing

Huang, Chin-Pao
Huang, Wu-Jang

Huang, Tony

Hummer, Daniel

Hunter, Timothy

Iglauer, Stefan

Ilankoon, Saman

İlhan, Sedat

Innocenzi, Valentina

Ippolito, Nicolò Maria

Ivanov, Maxim

Iveson, Alexander

Jackson, Togwell

Jacobs, Michael H.G.

Janots, Emilie

Jaszczak, John A.

Jiao, Yongqin

Jin, Jiaqi

Jo, Ho Young

Johnston, Karen E.

Jones, Franca

Jorand, Frédéric P.A.

Jordens, Adam

Jugo, Pedro

Jyothi, Rajesh Kumar

Kabelác, Martin

Kaduk, James

Kahl, Wolf-Achim

Kalaitzidis, Stavros

Kalbacova Vejpravova, Jana

Kalpaklı, Ahmet Orkun

Kamennaya, Nina

Kampmann, Tobias C.

Kanellopoulos, Christos

Kangal, Murat Olgaç

Kantzas, Apostolos

Kapsiotis, Argyrios

Karakashev, Stoyan I.

Karakus, Murat

Karayigit, Ali Ihsan

Kauko, Leiviskä

Kawano, Jun 
Kelm, Ursula

Khadilkar, Aditi

Khamkhash, Aibyek

Kim, Hyunjung

Kim, Hyeong-Ki

Kim, Yi-Yeoun

Kinnunen, Paivo

Kitagawa, Jiro

Klima, Mark

Ko, Ming-Sheng

Koenig, Alan

Kolker, Allan

Komljenovic, Dragan

König, Stephan

Kooyman, Patricia J.

Kornienko, Nikolay

Koshy, Pramod

Koutselas, Ioannis

Kovalenko, Galina A.

Kowalak, Stanisław

Kowalczuk, Przemyslaw

Kracht, Willy

Kremer, Barbara

Krivolutskaya, Nadezhda A.

Krivovichev, Vladimir

Kumar, Aloke

Kurniawan, Winarto

Kurt, Krenn

Kutschke, Sabine

Kuttner, Christian

Kynický, Jindřich

Kyzas, George Z.

Lanari, Pierre

Langley, Sean

Lao, Jonathan

Lascelles, Desmond

Laskowski, Janusz

Latifi, Nima

Laukamp, Carsten

Lavkulich, Les M.

Leary, Peter
Lecumberri-Sanchez, Pilar

Lee, Seung-Woo

Lee, Jaegeun

Lee, Joon Kyu

Lefticariu, Liliana

Lehto, Jukka

Lei, Qinghua

Leißner, Thomas

Lentz, David

León, Gerardo A.

Leou, Keh-Chyang

Lerouge, Catherine

Lesparre, Nolwenn

Leverington, David W.

Lewandowska, Anna

Lezzerini, Marco

Li, Hangyu

Li, Jian

Li, Chao

Lichtenegger, Helga

Lima, Selma M.

Liu, Wenying

Liu, Hongyuan

Liu, Yanan

Liu, Jia

Locock, Andrew

Lodge, Robert

Loredo, Jorge

Losada-Perez, Patricia

Lu, Liming

Luca, Raffaella

Lundström, Mari

Luptakova, Alena

Lüttge, Andreas

Ma, Tianshou

Machala, Libor

Mackinnon, Ian D.R.

Majka, Jarosław

Malaiškienè, Jurgita

Maldonado, Miguel

Malkovsky, Victor Ioannovich 
Mantovani, Luciana

Marini, Paola

Márquez, Gonzalo

Marsalek, Roman

Marta, Padoan

Martín-Crespo, Tomás

Martinetti, Alberto

Martínez, I.

Martín-Izard, Agustín

Martins, Tania

Masoumi, Hossein

Mateo-Lázaro, Jesús

Mathur, Ryan

Mavromatis, Vasileios

McBriarty, Martin

McDonald, Robbie

McFadzean, Belinda

Mclemore, Virginia $\mathrm{T}$.

McMaster, Scott

Medas, Daniela

Melchiorre, Erik

Melgarejo Draper, Joan Carles

Menad, Nourreddine Eddine

Menéndez-Aguado, J.M.

Mercier, Guy

Mesbah, Adel

Miki, Hajime

Milsch, Harald

Minniakhmetov, Ilnur

Miraglia, Salvatore

Mitra, Subhasish

Młynarczykowska, Anna

Molnar, Ferenc

Montanari, R.

Montero, José-María

Montorsi, Monia

Montoya, Alejandro

Moore, Moreton

Moreno-Maroto, José Manuel

Morishita, Tomoaki

Moxon, Terry
$\mathrm{Mu}$, Yufan

Mudd, Gavin

Mueller, Paul A.

Mueller, Ute

Mugnaioli, Enrico

Muppaneni, Tapaswy

Mustoe, George E.

Nagaraj, D.R.

Nagashima, Mariko

Najorka, Jens

Nanev, Christo

Natalicchio, Marcello

Neculita, Carmen Mihaela

Nejbert, Krzysztof

Newton, Aric

Nguyen, Nghiem Van

Ninomiya, Yoshihiko

Nishimura, Tatsuya

Noble, Aaron

Nowak, Jacek

Nowak, Pawel

Nuss, Philip

Obracaj, Dariusz

O'Connor, Louisa

Oggeri, Claudio

Oikonomopoulos, Ioannis

Ojovan, Michael

Okumura, Masahiko

Olea, Ricardo

Oliva, Josep

Omelon, Sidney

Önder, Mustafa

Ortega, J. Marcos

Ortiz, Julián

Orucoglu, Esra

Oshtrakh, Michael

Oskierski, Hans

Özcan, H. Kurtulus

Özkul, Mehmet

Palchik, Vyacheslav

Pan, Lei 
Pan, Shu-Yuan

Papastergios, Georgios

Parbhakar-Fox, Anita

Pardoen, Benoît

Park, Sol-Moi

Park, Cheon-Young

Pascual Martínez, Emilio

Pasquier, Louis-César

Pastero, Linda

Patterson, Joseph P.

Pavese, Alessandro

Pawlik, Marek

Pellicani, Roberta

Peng, Hong

Peng, Felicia

Pérez-Tello, Manuel

Peter, Wynn

Petrakis, Evangelos

Petranikova, Martina

Phillips, Steve

Picard, Donald

Pickles, Chris

Pieczka, Adam

Pierre, Francesco Dela

Pietrodangelo, Adriana

Pietrusiak, Damian

Piña, Rubén

Pingitore, Nicholas

Pironon, Jacques

Pita, Fernando

Placzek, Christa J.

Plante, Benoit

Pokrovsky, Oleg S.

Poli, Emiliano

Polini, Riccardo

Pollmann, Katrin

Pomi, Raffaella

Pontié, Maxime

Popov, Dmitry

Potra, Adriana

Pratt, Dyan
Précigout, Jacques

Předota, Milan

Prendes-Gero, Maria Belen

Pring, Allan

Prusek, Stanisław

Ptáček, Petr

Pulster, Erin

Putnis, Andrew

Putra, Heriansyah

Pyrcz, Michael

Qiu, Kun-Feng

Quirt, David

Ragozin, Alexey

Rahman, Md. Mujibur

Rao, Ashit

Raptopoulou, Catherine P.

Rassios, Annie Ewing

Rate, Andrew

Rddad, Larbi

Redler, Charlotte

Reed, David W.

Regueiro, Richard A.

Renforth, Phil

Renken, Albert

Renno, Axel D.

Retegan, Teodora

Rey-Raap, Natalia

Riegler, Thomas

Rimondi, Valentina

Rivoirard, Jacques

Rocha, Fernando T.F.T.

Rodehorst, Uta C.

Rodríguez, Gloria Belén Ramírez

Rodriguez-Navarro, Carlos

Rossana, Bellopede

Rout, Surya Snata

Rowles, Matthew R.

Royer, Jean-Jacques

Rudolph, Martin

Ruello, Maria Letizia

Ruuska, Jari 
Sabah, Eyüp

Sabrina, Hedrich

Sadeghi, Behnam

Saini-Eidukat, Bernhardt

Sakimoto, Kelsey

Sanchez-Jimenez, Pedro E.

Sand, Anders

Sanematsu, Kenzo

Santoro, Luciano

Santos, Rafael M.

Santos-Carballal, David

Sarver, Emily

Sassoni, Enrico

Saternus, Mariola

Saunders, James

Savik, Svein

Saydam, Serkan

Scala, Fabrizio

Schaeben, Helmut

Schatzel, Steven

Schindler, Michael

Schmidt, Jochen

Schobert, Harold

Scholz, Miklas

Schreiner, Marcus

Schwarcz, Henry

Schwarz, Phil

Scribano, Vittorio

Seifert, Soenke

Sejkora, Jiri

Shafiei, Ali

Shang, Julie Q.

Shen, Baotang

Shiers, Denis

Shin, Doyun

Shokuhfar, Tolou

Shuster, Jeremiah

$\mathrm{Si}$, Guangyao

Siedlecka, Ewa

Sierra-Fernández, Carlos

Silva, Z.C.
Sinche Gonzalez, Maria

Sindern, Sven

Sjöqvist, Axel

Skemer, Philip

Sklute, Elizabeth

Slatt, Roger M.

Slowakiewicz, Miroslaw

So, Jae-Seong

Sobianowska-Turek, Agnieszka

Song, Yungoo

Spagnoli, Dino

Spears, David Alan

Spiers, Christopher

Sprio, Simone

Steele-MacInnis, Matthew

Stefaniak, Paweł K.

Stoyanov, Stanislav R.

Strizhak, Pavel Alexandrovich

Stuart, Finlay

Su, Qinglin

Subías, Ignacio

Suorineni, Fidelis

Suryanto, Benny

Szczepaniak, Włodzimierz

Szczerba, Marek

Szilagyi, Robert

Taheri, Abbas

Tandlich, Roman

Tanner, Dominique

Tas, A. Cuneyt

Tavakoli, M.R.

Taxiarchou, Maria

Tayebi-Khorami, Maedeh

Temel, Halime Abakay

Tenorio, Victor Octavio

Thiéry, Vincent

Thomas, Rainer

Thompson, Jay

Tian, Feng

Timón, Vicente

Tischler, Judith 
Tobler, Dominique J.

Tom, Leistner

Torres Knoop, Ariana

Torró, Lisard

Torvela, Taija

Tuovinen, Olli

Turchyn, Sasha

Ueda, Takao

Ukar, Estibalitz

Ullmann, Clemens Vinzenz

Ulven, Ole Ivar

Urban, Petr

Vaccaro, Carmela

Valentim, Bruno

Vallianatos, Filippos

Van Den Kerkhof, Alfons M.

Van Der Meer, Freek

Van Driessche, Alexander

Varvara, Simona

Vasilatos, Charalampos

Vasyukova, Olga

Vaziri, Behad

Velasquez, German

Verdeja, Luis Felipe

Verrelli, David

Vicuña, Cristián Molina

Vieceli, Nathália

Villain, Sylvie

Vincenzi, Valentina

Visaveliya, Nikunjkumar

Vlieg, Elias

Vos, Cornelius Francois

Voudouris, Panagiotis

Wagner, Nicola

Wainwright, Alan

Wang, Gongwen

Wang, Yuhe

Wang, Hui

Wang, Vincent Z.

Wang, Zhipeng

Wang, Dengjun
Wang, Xuming

Wang, Zhongwei

Wang, Gang

Wang, Jianwei

Warszynski, Piotr

Watkin, Elizabeth

Webb, John A.

Webber, Grant

Pan, Wei-Ping

Weiss, Chester

Wells, Rachel

Wen, Xin

Wendler, Frank

Westaway, Robert

Whiteley, Andrew

Wilde, Andy

Wilkin, Richard

Winkelstern, Ian

Witt, Peter

Wohlgemuth-Ueberwasser, Cora

Wolf, Stephan E.

Wotruba, Hermann

Wragg, David S.

Wysokowski, Marcin

Xia, Fang

Xia, Liuyin

Xing, Huilin

Yahyaei, Mohsen

Yajima, Taro

Yamazaki, Tetsuo

Yanful, Ernest

Yang, Eric (Xue-Ming)

Yegulalp, Tuncel M.

Yilmaz, Erol

Yoo, Byung-Kuk

Yoon, Roe-Hoan

Yossifova, Mariana G.

Young, Courtney

Yu, Cheng-Ju

Yu, Changxun

Yue, Guikuan 
Yuk, Jong Min

Yvon, Jacques

Zawadzki, Jaroslaw

Zevenhoven, Ron

Zhang, Patrick

Zhang, Kuangyuan

Zhang, Wei

Zhang, Ruiyong

Zhang, Junfang

Zhang, Jinhong
Zhang, Guangqing

Zhang, Lejun

Zhou, Lingli

Zhou, Xuejun

Zhou, Z.A. (Joe)

Zhou, Binzhong

Zientek, Michael

Zimmermann, Robert

Zucali, Michele

(C) 2018 by the authors. Submitted for possible open access publication under the terms and conditions of the Creative Commons Attribution (CC-BY) license (http://creativecommons.org/licenses/by/4.0/). 